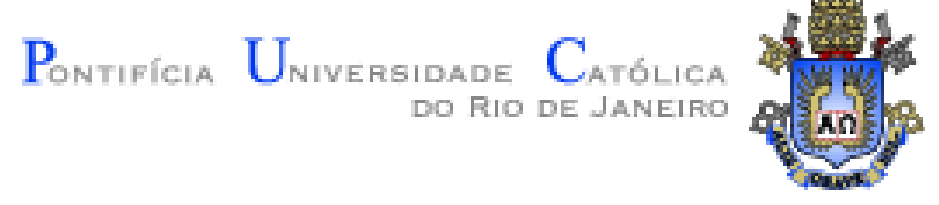

Frederico Rodrigues Abraham

\title{
Visualização de Modelos Massivos de \\ Reservatórios Naturais de Petróleo
}

Tese de Doutorado

Tese apresentada ao Programa de Pós-graduação em Informática do Departamento de Informática da PUC-Rio como requisito parcial para obtenção do título de Doutor em Informática

Orientador: Prof. Waldemar Celes Filho 


\title{
Visualização de Modelos Massivos de Reservatórios Naturais de Petróleo
}

Tese apresentada ao Programa de Pós-graduação em Informática do Departamento de Informática do Centro Técnico Científico da PUC-Rio como requisito parcial para obtenção do título de Doutor em Informática. Aprovada pela comissão examinadora abaixo assinada.

\author{
Prof. Waldemar Celes Filho \\ Orientador \\ Departamento de Informática - PUC-Rio \\ Prof. Alberto Barbosa Raposo \\ Departamento de Informática - PUC-Rio \\ Prof. Hélio Côrtes Vieira Lopes \\ Departamento de Matemática - PUC-Rio
}

Prof. Mario Costa Sousa

University of Calgary, Canadá

Prof. Claudio Esperança

Universidade Federal do Rio de Janeiro - UFRJ

Prof. José Eugênio Leal

Coordenador Setorial do Centro Técnico Científico - PUC-Rio 
Todos os direitos reservados. Proibida a reprodução total ou parcial do trabalho sem autorização da universidade, do autor e do orientador.

\section{Frederico Rodrigues Abraham}

Graduou-se em Engenharia da Computação na Pontifícia Universidade Católica do Rio de Janeiro. Fez uma iniciação científica no Departamento de Matemática, na qual desenvolveu softwares gráficos de auxílio ao ensino de Cálculo. Trabalhou na empresa Wiz em sistemas de telecomunicação sem fio. Desenvolveu ferramentas de visualização 3D com o grupo de visualização do laboratório Tecgraf durante o mestrado e o doutorado.

Ficha Catalográfica

Abraham, Frederico Rodrigues

Visualização de Modelos Massivos de Reservatórios Naturais de Petróleo / Frederico Rodrigues Abraham; orientador: Waldemar Celes Filho. - Rio de Janeiro : PUC-Rio, Departamento de Informática, 2011.

v., 122 f: il. ; $29,7 \mathrm{~cm}$

1. Tese (Doutorado em Informática) - Pontifícia Universidade Católica do Rio de Janeiro, Departamento de Informática.

Inclui referências bibliográficas.

1. Informática - Tese. 2. Renderização em Tempo Real. 3. Renderização Paralela. 4. Sistemas Distribuídos. 5. Estruturas de Multi-resolução. 6. Visualização com Níveis de Detalhe. 7. Visualização de Grandes Modelos. I. Celes Filho, Waldemar. II. Pontifícia Universidade Católica do Rio de Janeiro. Departamento de Informática. III. Título. 


\section{Agradecimentos}

Ao meu orientador Waldemar Celes Filho, pela paciência, disponibilidade e inspiração com que sempre me orientou durante o mestrado e o doutorado. Com ele, aprendi a encarar desafios com simplicidade, pragmatismo e objetividade. Além disso, é um dos melhores professores que já vi lecionar. É para mim uma grande influência.

Ao Tecgraf e aos colegas do Tecgraf: Andrei Monteiro, Antonio Calomeni, Márcia Earp, Marcio Pereira de Araujo, Paula Lucena, Renato Cerqueira, Rodrigo Espinha, Sérgio Maffra, Vitor Barata e Walter Habitzreuter.

Ao Professor Glaucio H. Paulino e ao NCSA (National Center for Supercomputing Applications) da Universidade de Illinois, EUA, pelo apoio e pelo acesso ao equipamento de cluster, sem o qual parte desta pesquisa não poderia ter sido concluída.

À minha querida Ana Paula Soares Carvalho, pelo amor, pelo carinho, pela paciência e pelo seu intelecto. A sua presença na minha vida foi fundamental, me inspirando e ajudando enormemente no meu crescimento pessoal e profissional.

À minha mãe Maria das Graças e aos meus amigos, pela alegria e pelos ensinamentos que sempre me deram.

Ao CNPq, à CAPES e à PUC-Rio, pelos auxílios concedidos, sem os quais este trabalho não poderia ter sido realizado. 


\title{
Resumo
}

\begin{abstract}
Abraham, Frederico Rodrigues; Celes Filho, Waldemar. Visualização de Modelos Massivos de Reservatórios Naturais de Petróleo. Rio de Janeiro, 2011. 122p. Tese de Doutorado - Departamento de Informática, Pontifícia Universidade Católica do Rio de Janeiro.
\end{abstract}

Os avanços recentes na tecnologia de simulação paralela de reservatórios de petróleo têm permitido a simulação numérica de domínios cada vez mais discretizados. Essas simulações produzem um volume de dados sem precedentes, que precisam ser visualizados em ambientes $3 \mathrm{D}$, possibilitando assim a análise e inspeção cuidadosa do modelo. Tais modelos tornam as técnicas convencionais de visualização inviáveis, criando a necessidade de se desenvolver soluções escaláveis de visualização. A necessidade de se visualizar dados tão complexos introduz diversos problemas computacionais que precisam ser tratados para visualizar o modelo com taxas de renderização interativas, como a impossibilidade de armazenar o dado todo em memória principal. Existem duas linhas principais para o tratamento de modelos de tal magnitude: renderização distribuída e técnicas de multi-resolução. Nesse trabalho são propostas soluções para a visualização de modelos massivos de reservatório de petróleo em cada uma dessas frentes de pesquisa, e é feita uma discussão acerca das vantagens e limitações de cada solução. Na primeira parte do trabalho, é proposto um sistema distribuído com ordenação no fim para a renderização de tais modelos em agrupamentos de PCs, onde cada PC é equipado com múltiplas GPUs. Dado o uso eficiente de cada GPU e de um estágio de composição parcial, nossa proposta trata dos problemas de escalabilidade que surgem em todo sistema com ordenação no fim em clusters de médio a grande porte. Na segunda parte do trabalho, é proposta uma estrutura hierárquica de multi-resolução de malhas de reservatórios de petróleo, com um novo algoritmo de simplificação feito especificamente para tais malhas. A estrutura hierárquica traz novidades em relação a trabalhos relacionados, fazendo uma estimativa de erro projetado menos conservadora. É feita uma proposta para a renderização com multi-resolução com garantia de uma taxa mínima de renderização, que é o objetivo principal de tais sistemas. Além disso, é feita uma proposta para o 
desenho do wireframe e das propriedades associadas à malha original do modelo de reservatório mapeados sobre as malhas simplificadas, o que torna a estrutura de multi-resolução independente das propriedades geradas pela simulação, garantindo o seu reuso ao longo de múltiplas simulações do mesmo modelo. Nossos experimentos computacionais demonstram a eficiência das soluções propostas.

\section{Palavras-chave}

Renderização em Tempo Real ; Renderização Paralela ; Sistemas Distribuídos ; Estruturas de Multi-resolução ; Visualização com Níveis de Detalhe ; Visualização de Grandes Modelos . 


\section{Abstract}

Abraham, Frederico Rodrigues; Celes Filho, Waldemar (advisor). Visualization of Complex Natural Black Oil Reservoir Models. Rio de Janeiro, 2011. 122p. DSc Thesis — Departamento de Informática, Pontifícia Universidade Católica do Rio de Janeiro.

Recent advances in parallel architectures for the numerical simulation of natural black oil reservoirs have allowed the simulation of very discretized domains. As a consequence, these simulations produce an unprecedented volume of data, which must be visualized in 3D environments for careful analysis and inspection of the model. Conventional scientific visualization techniques of such very large models are not viable, creating a demand for the development of scalable visualization solutions. The need for the visualization of such complex data introduces several computational issues which must be addressed in order to achieve interactive rendering rates, such as the impossibility of storing the entire data in main memory. There are two main research areas which propose solutions for the visualization of models with such magnitude: distributed rendering and multi-resolution techniques. This work proposes solutions for the visualization of massively complex reservoir models in each of these research areas, and a discussion over the advantages and limitations of each solution is made. In the first part of the work, we propose a distributed system based on a sort-last approach for the rendering of such models in PC clusters, where each PC is equipped with multiple GPUs. Given an efficient use of the available GPUs, combined with a pipelined implementation and the use of partial image compositions on the cluster nodes, our proposal tackles the scalability issues that arise when using mid-to-large GPU clusters. The second part of the work brings the proposal of a hierarchical multi-resolution structure of black oil reservoir meshes, with a new simplification algorithm designed specifically for such meshes. The hierarchical structure brings some new approaches in relation to related work, doing a much less conservative projected error estimation. We propose a minimum refresh rate guarantee strategy for our multiresolution rendering, which is the main goal for such systems. Afterwards, we introduce a proposal for the rendering of data associated with the original 
reservoir mesh mapped over the simplified meshes, such as the original model grid wireframe and reservoir properties. This proposal guarantees an independence between the multi-resolution structure and the properties generated by a simulation, which guarantees the reuse of the structure among several simulations of the same model. Experimental results demonstrate the effectiveness of the proposed solutions.

\section{Keywords}

Real-time Rendering ; Parallel Rendering ; Distributed Systems ; Multiresolution Data Structures ; Visualization with Levels of Detail ; Massive Model Visualization . 


\section{Sumário}

1 Introdução $\quad 11$

$\begin{array}{lll}1.1 & \text { Contribuições } & 15\end{array}$

$\begin{array}{lll}1.2 & \text { Organização do Documento } & 17\end{array}$

2 Trabalhos Relacionados $\quad 18$

2.1 Técnicas de Descarte de Primitivas Gráficas 18

$\begin{array}{ll}2.2 \text { Visualização Distribuída } & 19\end{array}$

2.3 Visualização com Níveis de Detalhe e Multiresolução 21

3 Modelo de Reservatórios Naturais de Petróleo $\quad 27$

3.1 Simulação de Reservatórios $\quad 27$

3.2 Modelos de Reservatório 28

3.3 Visualização de Modelos de Reservatório 30

3.4 Visualização de Modelos de Médio Porte 31

4 Visualização Distribuída $\quad 37$

$\begin{array}{lll}4.1 & \text { Arquitetura de Hardware } & 38\end{array}$

4.2 Partição do Modelo 38

4.3 Linha de Produção Distribuída 40

4.4 Resultados Experimentais 43

5 Visualização com Multi-resolução $\quad 48$

5.1 Simplificação de Malhas de Reservatório 48

5.2 Hierarquia de Multi-resolução $\quad 57$

$\begin{array}{ll}5.3 \text { Malhas para Visualização } & 60\end{array}$

5.4 Visualização Dependente do Observador 63

$\begin{array}{lll}5.5 & \text { Resultados Experimentais } & 70\end{array}$

$6 \quad$ Mapeamento de Propriedades $\quad 85$

6.1 Mapeamento do Grid Original $\quad 86$

6.2 Mapeamento de Propriedades $\quad 93$

$\begin{array}{ll}6.3 \text { Resultado Experimental } & 110\end{array}$

$\begin{array}{ll}6.4 \text { Conclusão } & 111\end{array}$

7 Conclusão e Trabalhos Futuros $\quad 113$

7.1 Visualização Distribuída 113

7.2 Visualização com Multi-resolução 114

7.3 Mapeamento de Propriedades 115

8 Referências Bibliográficas $\quad 117$ 
La semplicità costituisce l'ultima sofisticazione.

Em português: A simplicidade é a sofisticação final.

Frase atribuída a Leonardo da Vinci. 